\title{
BLAST Application on the GPE/UnicoreGS Grid
}

\author{
Marcelina Borcz, Rafał Kluszczyński, and Piotr Bała \\ Nicolaus Copernicus University, \\ Faculty of Mathematics and Computer Science, \\ ul. Chopina 12/18, 87-100 Toruń, Poland \\ \{marbor,klusi, bala\}@mat.uni.torun.pl
}

\begin{abstract}
Sequence analysis is one of the most fundamental tasks in molecular biology. Because of the increasing number of sequences we still need more computing power. One of the solutions are grid environments, which make use of computing centers. In this paper we present plug-in which enables the use of BLAST software for sequence analysis within Grid environments such as UNICORE (Uniform Interface to Computing Resources) and GPE (Grid Programming Environment).
\end{abstract}

\section{Introduction}

Grid technology is becoming very popular nowadays. Watching weather forecast we usually do not even realize how much computing power has been used to prepare it. Scientific research and technology development implies an increasing demand for distributed resources. We still need more precise weather forecast, longer molecular simulations and we want to determine genome structure and its functions. In other words, we need computing power which could easily be made available to the user by means of Grid middleware like UNICORE [16] or Globus 9.

The concept of Grid computing was first introduced in 1999 [7. Its main idea is the use of multiple distributed resources combined together on a single application to work cooperatively. Definition of Grid computing have evolved during the time [8]. Ian Foster in [6] suggested simple checklist definition of the Grid technology composed of 3 points:

- The resources it uses should be distributed without any centralized control.

- All protocols used for authorization, resource discovery etc. should be open and standard.

- The use of distributed resources in combined form should be much more worthwhile than using them separately.

Grid middlewares find the use in much of scientific research. They have been successfully used in 3D graphics, quantum chemistry and molecular modeling. Since it became possible to determine the structure and sequence of DNA, many scientists have been trying to indicate the role of specific DNA motifs in human

W. Lehner et al. (Eds.): Euro-Par 2006 Workshops, LNCS 4375, pp. 245-253, 2007.

(C) Springer-Verlag Berlin Heidelberg 2007 
organism. This gives the opportunity to model many biological processes in our body. It may also help us to cure many diseases, for example by blocking expressions of specific genes. Since Basic Local Alignment Search Tool (BLAST) appeared [1, it has become one of the fundamental and widely used tools in molecular biology for sequence analysis. Prediction of human metabolic pathways can serve as an example of using BLAST to find human enzymes taking part in metabolic process (see [15]). Since 1990 we can observe an increasing need for the use of the BLAST application by molecular biology scientists. That is why there have been many attempts to optimize and speed up this biological tool. For example in 1998 in [5] there were presented modifications which produce a better suited code for comparing large numbers of sequence to several different

databases. Grid middleware brings another way of comparing a large number of sequences. In a simple way comparisons of different sequences can be distributed within different computing centers where original BLAST code can be used. This eliminates errors which could occur during applying proposed modifications to the code. The purpose of this paper is to show Grid technology solution by presenting plug-in for BLAST application designed for UNICORE and GPE middleware.

\section{BLAST Software}

The Central Dogma of Molecular Biology states, that two-stranded helix of DNA composed of four types of nucleotides, is duplicated during replication process. Next, DNA is transcribed into one-stranded structure of RNA. Finally protein, i.e. polymers built from 20 different types of amino-acids, is synthesized from RNA by translation. Sequences can change over time due to mutation, natural selection and genetic drift. By means of BLAST biologists are able to compare DNA or protein sequences from the same or different organisms. In this way evolutionary relationship between organisms can be explored and biological functions of new sequences can be predicted.

BLAST (Basic Local Alignment Search Tool) became an important and the most widely used tool in the field of bioinformatics. It finds statistically significant local similarities between pairs: user-defined (protein or DNA) sequence and sequences from databases. As an output it gives gapped or gap-free alignments. Each alignment is a high-scoring segment pair with final score and estimation of statistical significance, called E-value (see [2] for details). During DNA sequences comparison, results contain also information about DNA strand direction where the similarity has been found.

The popularity of the program, apart from its functionality, is based on the programs following qualities: reliability, speed and flexibility. BLAST is the name of a package of software containing, among other things, blastp and blastn programs (see 3] for detailed description). Each of these components realizes algorithms for different types of sequences (blastp for proteins, blastn for nucleotides). BLAST uses a heuristic algorithm. First it takes subsequences of query sentence of fixed length and all words with the same length which alignment score is at 
least the pre-defined threshold value. Next, database is scanned for these words called "hot spots", which are extended in both directions until some threshold or cut-off value is reached. In this way BLAST finds similarities without exploring the entire search space which could take a lot of time.

There are also several variants of BLAST, such as PSI-BLAST (PositionSpecific Iterated BLAST) or PHI-BLAST (Pattern-Hit Initiated BLAST), which run specialized version of BLAST programs. First of them uses PSSMs (PositionSpecific Scoring Matrices) to improve sensitivity of searching. In the second program, there can be put a motif next to the query sequence, which is the most significant and is used to scan the database for, instead for the "hot spots".

BLAST software is being developed by many institutes. The most popular versions of the programs are NCBI-BLAST and WU-BLAST. The first of them originated from the National Center for Biotechnology Information, while the other is being developed at Washington University in St. Louis. Each version has plenty of options which makes BLAST a very flexible tool used by biologists all over the world.

\section{Grid Programming Environment (GPE)}

Grid environments are rapidly changing because of the new standards and application areas which are still emerging and are being discovered. That is why Grid Programming Environment (GPE) is being currently developed. The goal is to implement new grid middleware based on the experience gained during the implementation of UNICORE. Because the success of the Grid technology depends among others on interoperability between different Grid implementations, there is an idea to establish a stable interface between them. It is also important to build a more flexible and user-friendly client framework for heterogeneous Grid infrastructures with easy and secure access.

With the concept of GPE project (see [14] for more details) comes different client applications designed for three category of users:

- Expert Users - with the ability to construct complex workflows of their jobs (it is the successor of the UNICORE Client). This type of client is dedicated to users with some knowledge about the functionality of the Grid. Besides the workflow editor, the user has the ability to use different identities on different target systems.

- Application Users - it is sufficient for them to use only one application, so they do not need all of the tools available in order to build workflows. This client interface doesn't have all the functionality offered by the Grid. Instead of that it is very easy and intuitive to use. Application Client depicted in Fig. 1 is dedicated to scientists who usually run only one application at the time.

- Unaware Users who manage their tasks and Grid resources through portals in web browsers. Such users do not even have to install client application. 


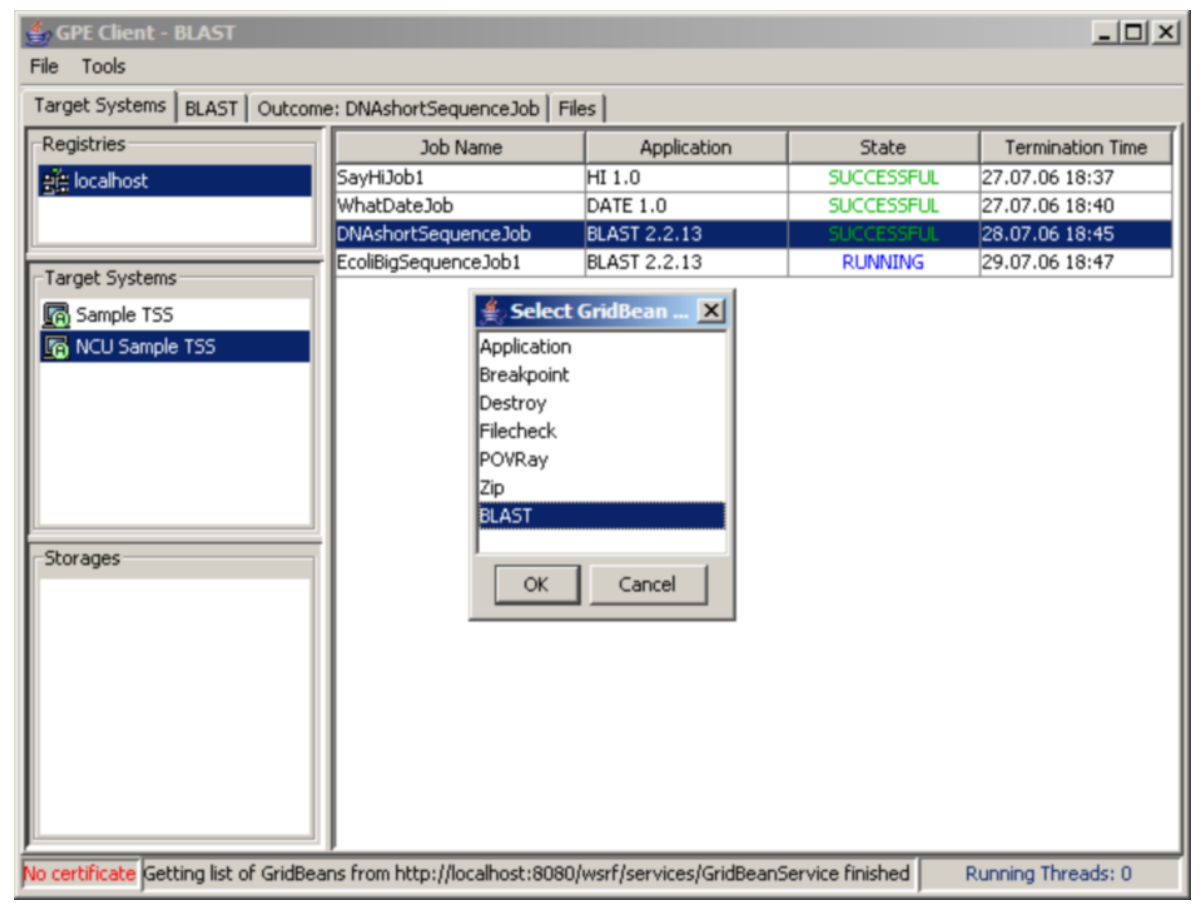

Fig. 1. GPE client interface dedicated for application users. It can be seen response from GridBean service presenting list of available plug-ins on specified target system.

Everything they need on the computer is just an Internet browser. Such a solution is also very attractive for mobile users who could manage their tasks from any Internet cafe.

GridBean approach is one of the major advantages of the GPE framework. Usually, when user wants to use specific application on the grid, he has to download corresponding plug-in and integrate it with the client program. Sometimes specific knowledge about the structure of the software is needed, like directory name where the plug-in files should be placed. However, not every user has some knowledge about computer science, even the basic one. That is why the concept of GridBeans is very promising. Instead of downloading and manually integrating the needed plug-in, GPE client software has the ability to check what applications are available on the remote system. In the case the list contains the application the user wants to work with, it is enough to select it by clicking resulting in the corresponding plug-in being automatically downloaded into the client's job managing interface and made ready to use.

In other words, GridBeans are plug-ins which can be loaded dynamically into any of the three different client applications. There is no need to have any detailed knowledge about the physical structure of the software in order to use 
available application. Ratering in [14] described four major advantages standing for presented GridBean approach:

- Easy distribution and update of plug-ins - updating plug-ins takes place in the same way like downloading. User has to ask GridBean service for available gridbeans and then downloads new version of plug-in he is interested in.

- Overview of supported applications - once the user asks the GridBean service for plug-ins available on the target system, he gets clearly presented list of actually supported applications.

- Flexibility of GridBeans - once the plug-in has been implemented, there is no need to create different copies for different types of client applications discussed above. Special care may be only needed for graphical layout depends on the type of client.

- Easy implementation of GridBeans - this is especially important for developers bringing applications on the Grid. The main effort to design plug-in is implementation of the graphical interface and construction of the job description. Thanks to recipe guide [1], even non-professional programmer will be able to design plug-in in reasonable time.

As it was mentioned, these points make the GridBean approach very promising. Thanks to inter-operating GridBean service and client programs, manipulating with plug-ins for different applications has become very easy for the user, even one without any computer science background.

Grid Programming Environment was designed based on the experience of UNICORE implementation. To keep track of all resources available on all target systems Target System Registry has been created. Every target system during start-up registers its resources properties as installed software, workload or running jobs. This information is being dynamically updated during runtime by Target System Service. Presented solution assures that when client asks for available resources he gets always up-to-date information. Target System Registry, besides managing the list of available target systems with their resources, plays another crucial role. In similar way to the classical UNICORE Gateway, it performs authentication to prevent any access to the services from unsafe Internet.

The GPE Clients can contact to the Target Systems Services available in the different hosting environments such as Globus or UnicoreGS. In result, it is possible to develop interface which can be used with the different middleware without any modifications.

\section{BLAST GridBean for GPE}

In this section we present GridBean designed for BLAST application. User interface is organized in a similar way to the one on NCBI-BLAST website [12]. The main reason of such design is to keep it easy to use, specially by scientists who are used to NCBI website and could have some troubles to migrate on the GPE 


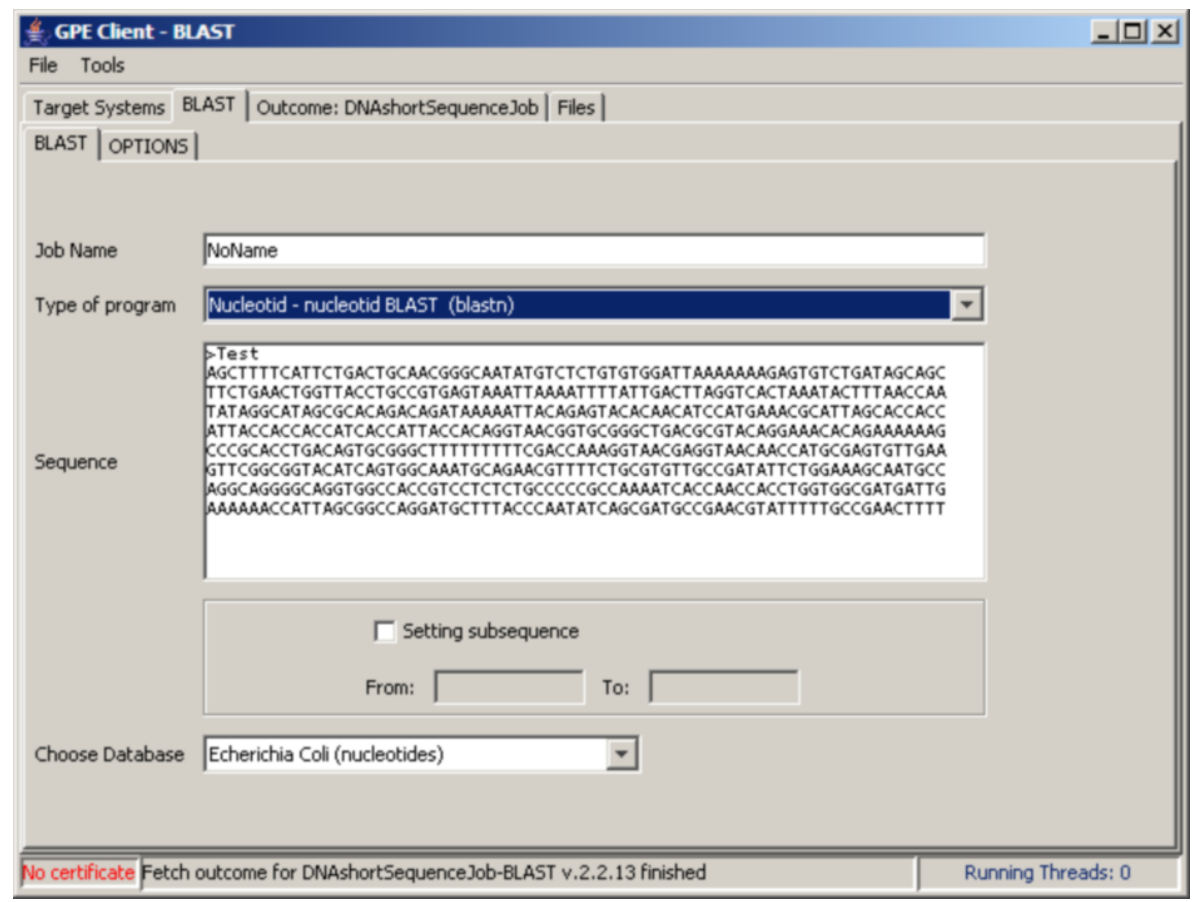

Fig. 2. Main graphical user interface panel. It grants a choice of the type of BLAST program and a possibility to enter the sequence and to specify a database which is to be browsed.

framework. In the main panel, besides the job name, a particular sequence can be entered so that it may be compared with the sequences from a chosen database. There can be typed either a whole sequence or the definition line only (Fig. 2). There also should be chosen the type of BLAST program and the database that the sequence will be search in.

The second panel provides optional choices which affect the presentation of the search results. In the case of using blastp program user should determine which scoring matrix he wants to use. Accuracy of matching can be controlled by changing appropriately expect and word size values (Fig. 31). There is also a possibility to determine gap penalties for protein comparison. Flexibility of searching similarities can be increased by using filters while readability of output by choosing alignment view and convenient number of descriptions and alignments to be displayed. In the last field of the panel advanced user can enter more sophisticated and more rarely used options, like "-I T" which show GenInfo Identifier number in definition lines.

Presented panels (Input Panels) are extensions of the GridBean panel designed for inputing the data by the user. Input Panel provides a visual area to display needed parameter controls, and also a technique enabling the correlation of values gained from those controls with the job description. Depending 


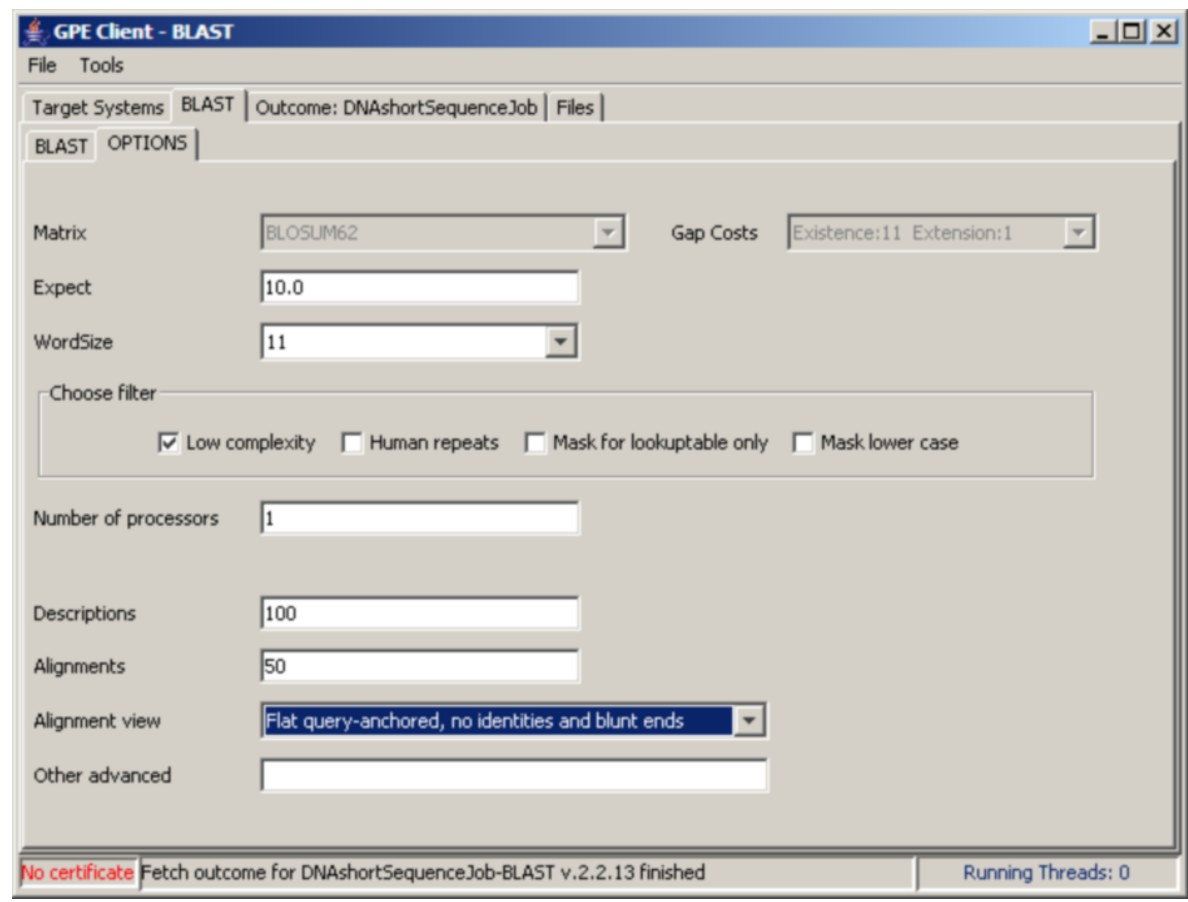

Fig. 3. Second panel of client's GUI. Here the user specifies more detailed data including the number of similar sequences or alignments to be show. It is also possible to enter some specific options for BLAST package expert users.

on the application and its complexity, which is usually determined by a large number of parameters, there is a possibility of using more than one input panel for grouping the varied options respectively. Another advantage determining the flexibility of the Gridbean approach is, that the plug-in can also have Output Panels. Their main purpose is to display the job results downloaded from the Grid, but the way of presenting the data can be made much more attractive and more intuitive than the raw text. It could for instance show some precomputed graphics, charts or tables with statistics. Of course, designing such panels would strictly be connected with the application the GridBean is meant to be used with. It is therefore recommended for the developers to have a good knowledge of the software or a good specialist nearby to address any questions. It is also important to mention that thanks to Grid Services the configuration of available databases for BLAST can be made on the Target System. Such solution ensures that there is no need to upgrade the GridBean after changing the database list on the server. BLAST plug-in simply checks the list before the user starts preparing the task. This means that the user can see only those databases which are currently available in the system. 
The authors have successfully designed and implemented an interface for BLAST application to run on the Grids. This plug-in, without any modifications, can be also used as an element of more sophisticated workflow tasks. Building that kind of tasks will be possible with Expert Client application. The GridBean implementation was developed under the GPE4GTK project [10] and has been tested with the binary distribution, called GPE-Lite (release 1.0.0), available at SourceForge website 13 . For testing the BLAST application has also been used (version 2.2.13) available at the NCBI website [12].

\section{Conclusions and Future Work}

In this paper we have presented plug-in designed for being currently developed GPE framework. BLAST GridBean will allow many bioinformatic scientists to use Grid technology instead of usually overloaded NCBI servers. Another advantage of Grid middleware, that we have mentioned above, is easy implementation of plug-ins. All the developers have to worry about is the graphic interface displayed to the user and job specification to run on computing center's side. Moreover it is easy to extend standard results presentation which usually is in text format. Based on this output data we can extract information to present it in specific graphical way. Indeed, it is the object of our current work to add graphical presentation of BLAST application results (i.e. alignments) using BioJava package [4].

\section{References}

1. Altschul, S., Gish, W., Miller, W., Myers, E.W., Lipman, D.: A Basic Local Alignment Search Tool. Journal of Molecular Biology 215:403-410 (1990).

2. Altschul, S., Karlin, S.: Applications and Statistics for Multiple High-Scoring Segments in Molecular Sequences. Proceedings of the National Academy of Sciences 90:5873-5877 (1993).

3. Bedell, J., Korf, I., Yandell, M.: BLAST. O'Reilly \& Associates (2003).

4. BioJava project website: http://www.biojava.org/wiki/Main_Page.

5. Camp, N., Cofer, H., Gomperts, R.: High-Throughput BLAST. SGI White Paper (1998).

6. Foster, I.: What is the Grid? A Three Point Checklist. Grid Today, Vol. 1(6), Argonne National Laboratory \& University of Chicago (2002). Available at: http://www. gridtoday. com/02/0722/100136.html.

7. Foster, I., Kesselman, C. (Eds.): The Grid: Blueprint for a New Computing Infrastructure. Morgan Kaufmann Publishers (1999).

8. Foster, I., Kesselman, C., Tuecke, S.: The Anatomy of the Grid, Enabling Scalable Virtual Organizations. International Journal of Supercomputer Applications, 15(3):200-222 (2001).

9. Globus project website: http://www.globus.org.

10. GPE4GTK project website: http://gpe4gtk. sourceforge.net. 
11. GridBean Cookbook. UniGrids project documentation (2005).

12. NCBI BLAST website: http://www.ncbi.nih.gov/BLAST.

13. Open Source projects website: http://sourceforge.net.

14. Ratering, R.: Grid Programming Environment (GPE) Concepts. Intel Corporation, GPE documentation (2005).

15. Romero, P., Wagg, J., Green, M.L., Kaiser, D., Krummenacker M., Karp, P.D.: Computational prediction of human metabolic pathways from the complete human genome. Genome Biology 6:R2 (2004).

16. UNICORE project website: http://unicore.sourceforge.net. 